\title{
Towards Accurate Shape Reconstruction of Compact Bionic Handling Arm
}

\author{
Inderjeet Singh ${ }^{1}$, Yacine Amara ${ }^{2}$, Manarshhjot Singh ${ }^{1}$ and Rochdi Merzouki ${ }^{1}$
}

\begin{abstract}
Continuum robots can continuously curve to give much more flexibility than rigid robots. Usually, continuum robots are made up of soft flexible material and have complex structures to provide high dexterity of motion. The presence of these factors, i.e., soft material and complex structure, make mathematical modeling of continuum robots a cumbersome task. This paper aims to discuss the Pythagorean Hodograph (PH) based method for shape reconstruction of continuum robots and proposes a methodology for improving this reconstructed shape using experimental data. The method is successfully applied to Compact Bionic Handling Arm (CBHA) manipulator. The results show a clear improvement in the accuracy.
\end{abstract}

\section{INTRODUCTION}

Continuum robots can mimic the behavior of biological mobalizers like an elephant's trunk [1], a fish or an octopus [2], due to their soft material. Modeling them is difficult due to the non-linearity and high degrees of freedom in their structure.

Continuum manipulators are being studied for their kinematics and shape reconstruction. Bionic Handling arm (BHA) manipulator is modeled kinematically by representing it as torus segments [3]. Different forward kinematic models (FKMs) for CBHA manipulator are discussed and also compared regarding accuracies in [4]. A combination of optimization and pattern recognition is used to find optimal setting for multiple CBHAs in parallel configuration in [5]. In [6], a geometrical model is developed for OctArm V manipulator using cosserat rod theory and a fiber reinforced model. Inverse kinematic model (IKM) of a continuum manipulator is described using feed-forward neural networks in [7]. In [8], various IKM models (Hybrid approach, Neural Network approach, Newton Raphson approach and Damped least square approach) for CBHA manipulator are compared on the basis of accuracy and time cost.

Furthermore, in case of shape reconstruction of continuum manipulators, sensors are used in most of the works [9], [10], [11]. In [12], a correspondence between a hyper-redundant manipulator and a geometric curve has been established by solving a non-linear optimization problem called 'shape inverse problem'. The backbone shape of a hyper-redundant manipulator has also been solved using the modal approach and thereby using a fitting algorithm to join the various

1 I. Singh, M. Singh and R. Merzouki are with Polytech Lille, CRIStAL, CNRS-UMR 9189, Avenue Paul Langevin, 59655 Villeneuve d'Ascq, France inderjeet.singheed.univ-lillel.fr manarshhjot.singhegmail.com

rochdi.merzouki@polytech-lille.fr

${ }^{2}$ Y. Amara is with Ecole Militaire Polytechnique, BP 17, Bordj EL Bahri, Algiers 16111, Algeria amara.yacinedgmail.com sections of a discrete 30 DOF manipulator [13]. Pythagorean Hodograph curves and their applications are defined by Farouki and Sakkalis in 1990 [14]. These curves have the following main properties: 1) Length as well as bending energy of these curves can be calculated in the closed form 2) Easy to construct parallel curves 3) Only four boundary conditions are required to construct a quintic PH curve i.e. starting position, final position and direction vectors at both of these positions. In the field of robotics, $\mathrm{PH}$ curves are used mainly for the trajectory planning purposes. $\mathrm{PH}$ curves have been used for 3D path planning of three unmanned aerial vehicles (UAVs), as well as to avoid obstacles in their path [15]. In [16], PH curves are being utilized for optimal path planning of mobile robots and also the same concept is introduced for the calculation of backbone curve of a two-dimensional hyper-redundant manipulator. In [17], PH curves are utilized to model spacial continuum manipulators, which is being extended in this paper. This paper focuses on improving the accuracy of the $\mathrm{PH}$ based reconstructed shape for CBHA manipulator. The main contributions of this work are:

- Discussion of shape reconstruction of CBHA manipulator using PH curves based approach. Improvement in the accuracy of the reconstructed shape using experimental data.

- A relation is developed between existing PH based shape and corrected shape using neural networks approach.

The organization of the paper is as follows: Section II gives a brief description of the CBHA manipulator. Section III describes the Pythagorean hodograph condition and also the generation of the PH curves. Section IV introduces the methodology used for improving the accuracy of the shape for the CBHA manipulator. Results and discussions are presented in section V. Finally, the conclusions, as well as future perspectives of the presented work, are discussed in section VI.

\section{DeSCRIPTION OF CBHA MANIPUlator}

CBHA manipulator, also called elephant trunk manipulator, is made up of a soft elastic polyamide material, where it is divided into two sections, a rotating wrist and a compliant gripper (Fig. 1). Each section is composed of three tubes, connected each other through a backbone, and actuated by electro-pneumatic actuators. Pressure change inside the tubes leads to change in their respective lengths, inducing the change in the position and orientation of the tip of the manipulator. 


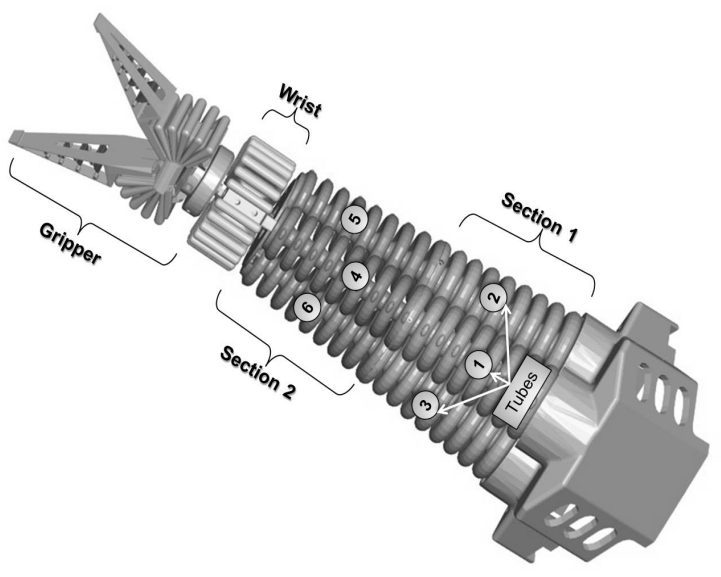

Fig. 1. CBHA manipulator description

Equal pressures in all three tubes maintain the robot in straight line longitudinal posture. An inextensible cable placed at the backbone of the manipulator limits the maximum extension. To create bending, differential pressures can be applied inside the tubes. The elongation of each tube is measured with a wire-potentiometer. There are six wirepotentiometers, three of them measure the tube lengths of the first section, and the three others measure the total tube lengths.

\section{Pythagorean Hodograph In Context of \\ SHAPE RECONSTRUCTION OF CBHA MANIPULATOR}

In the following development, it is supposed that the two bending sections (Fig. 1) are fused at a junction point, to form one entire continuum section, without any shape disconnection. Pythagorean Hodograph curves are used in the context of CBHA manipulator to reconstruct its optimal posture [17]. Let us consider two spacial poses $P_{s}$ and $P_{f}$ on the CBHA manipulator as:

$$
\begin{array}{r}
P_{s}\left(x_{s}, y_{s}, z_{s}, \alpha_{s}, \beta_{s}, \gamma_{s}\right) \\
P_{f}\left(x_{f}, y_{f}, z_{f}, \alpha_{f}, \beta_{f}, \gamma_{f}\right)
\end{array}
$$

Here, $P_{s}$ is the starting pose (position $x_{s}, y_{s}, z_{s}$ and orientation $\left.\alpha_{s}, \beta_{s}, \gamma_{s}\right)$ of the CBHA manipulator located at its base. $P_{f}$ is the final pose (position $x_{f}, y_{f}, z_{f}$ and orientation $\alpha_{f}, \beta_{f}, \gamma_{f}$ ) of the CBHA manipulator located at its end. PH curve is used to reconstruct the shape of the manipulator from its starting pose to the final pose. PH curves are the normal polynomial curves with an extra condition of Pythagorean Hodograph. Therefore, these curves are the special case of the normal polynomial curves and are explained as follows [18]:

Let $r(h)$ be the normal polynomial curve used to reconstruct the shape of the CBHA manipulator.

$$
r(h)=(x(h), y(h), z(h)) ; \quad 0 \leq h \leq 1
$$

$h$ be the normalized curvilinear coordinate of the curve. It means:

$$
\begin{aligned}
P_{s} & =r(0)=(x(0), y(0), z(0)) \\
P_{f} & =r(1)=(x(1), y(1), z(1))
\end{aligned}
$$

Hodograph is a term defined as the first derivative of the curve $r(h)$. It is parallel to the tangent to the curve. It is given as:

$$
r^{\prime}(h)=\left(x^{\prime}(h), y^{\prime}(h), z^{\prime}(h)\right)
$$

The length $L(h)$ of the CBHA manipulator reconstructed using curve $r(h)$ is:

$$
L(h)=\int_{0}^{1}\left|r^{\prime}(h)\right| \mathrm{d} h=\int_{0}^{1} \sqrt{x^{\prime}(h)^{2}+y^{\prime}(h)^{2}+z^{\prime}(h)^{2}} \mathrm{~d} h
$$

Equation (5) represents the length of the CBHA manipulator. Due to the presence of the square root in the integral, the closed form solution of the length is not possible. Therefore, a numerical method is required to find the approximation of the actual solution. To overcome this situation, the square root sign can be replaced, if:

$$
x^{\prime}(h)^{2}+y^{\prime}(h)^{2}+z^{\prime}(h)^{2}=\sigma(h)^{2}
$$

This is called the Pythagorean law or condition in three dimensions. Therefore,

The first derivatives (Hodographs) of parametric polynomials which satisfy the Pythagorean condition, are known as Pythagorean Hodographs.

Now length of the CBHA manipulator can be calculated in the closed form as:

$$
L(h)=\int_{0}^{1}|\sigma(h)| \mathrm{d} h
$$

A sufficient and necessary condition which satisfies eq. (6) is given in [19], [18], i.e. the four polynomials $u(h), v(h), p(h)$ and $q(h)$ are used to express the hodograph components as:

$$
\begin{gathered}
x^{\prime}(h)=\left[u^{2}(h)+v^{2}(h)-p^{2}(h)-q^{2}(h)\right] \\
y^{\prime}(h)=2[u(h) q(h)+v(h) p(h)] \\
z^{\prime}(h)=2[v(h) q(h)-u(h) p(h)]
\end{gathered}
$$

$u(h), v(h), p(h)$ and $q(h)$ polynomials are chosen so that $\sigma(h)$ is:

$$
\sigma(h)=u^{2}(h)+v^{2}(h)+p^{2}(h)+q^{2}(h)
$$

Generalized degree of $\mathrm{PH}$ curves is $2 n+1$, where $n$ is the degree of polynomials $u(h), v(h), p(h)$ and $q(h)$. So, the lowest degree $\mathrm{PH}$ curves are cubic PH curves when linear polynomials are used. Cubic $\mathrm{PH}$ curve does not have any free control point (out of four control points), it means they have a unique solution only. Therefore, cubic PH curves are not good for shape representation. Quintic PH curves can be generated by using quadratic polynomials $u(h), v(h), p(h)$ and $q(h)$. Quintic PH curves contain two free control points which makes them more suitable to reconstruct the actual shapes accurately [18]. Hence, the following development uses the quintic PH curves to model CBHA manipulator. The expression of quintic $\mathrm{PH}$ curve is derived by using quadratic polynomials $u(h), v(h), p(h)$ and $q(h)$ in the Bernstein form [20], [18]. These quadratic polynomials are inserted in eqs. 
$(8,9,10)$ and integrated from both sides to yield the general form of quintic $\mathrm{PH}$ curve $r(h)$ :

$$
r(h)=\left[\begin{array}{l}
x(h) \\
y(h) \\
z(h)
\end{array}\right]=\sum_{k=0}^{5} P_{k}\left(\begin{array}{l}
5 \\
k
\end{array}\right)(1-h)^{5-k} h^{k}
$$

It implies,

$$
r(h)=\left[\begin{array}{c}
1 \\
h \\
h^{2} \\
h^{3} \\
h^{4} \\
h^{5}
\end{array}\right]^{T}\left[\begin{array}{cccccc}
1 & 0 & 0 & 0 & 0 & 0 \\
-5 & 5 & 0 & 0 & 0 & 0 \\
10 & -20 & 10 & 0 & 0 & 0 \\
-10 & 30 & -30 & 10 & 0 & 0 \\
5 & -20 & 30 & -20 & 5 & 0 \\
-1 & 5 & -10 & 10 & -5 & 1
\end{array}\right]\left[\begin{array}{c}
P_{0} \\
P_{1} \\
P_{2} \\
P_{3} \\
P_{4} \\
P_{5}
\end{array}\right]
$$

The derived form is the Bernstein-Bezier form. Here, $P_{k}=$ $\left(x_{k}, y_{k}, z_{k}\right)$ are the control points. These control points are in terms of $u(h), v(h), p(h)$ and $q(h)$. Therefore next step is to find these optimal polynomials $u(h), v(h), p(h)$ and $q(h)$ to calculate the control points $P_{k}$.

Basic Schematic of a quintic $\mathrm{PH}$ curve to reconstruct the

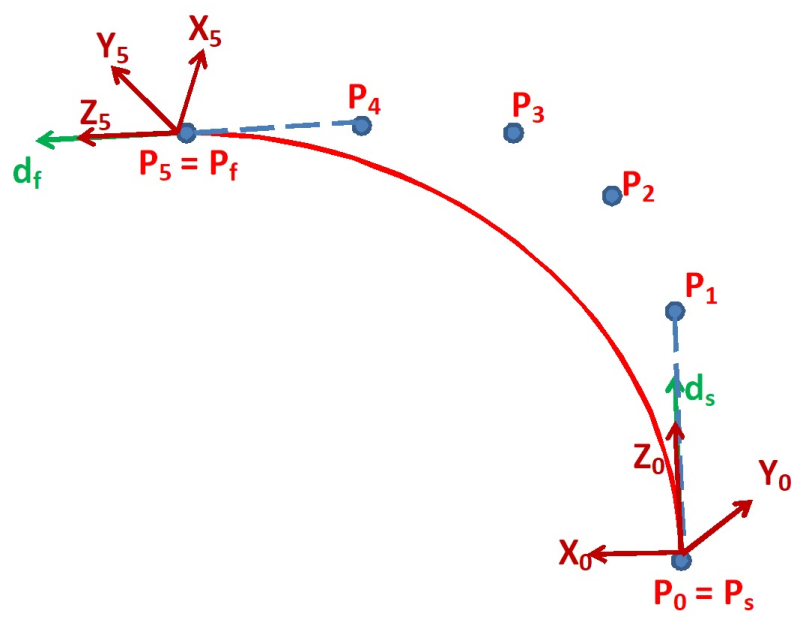

Fig. 2. Basic schematic of PH curve for CBHA manipulator

shape of the CBHA manipulator is shown in Fig. 2. $\vec{d}_{s}$ and $\vec{d}_{f}$ are the direction vectors at starting (base) and ending point of CBHA manipulator respectively. They can be computed using orientations $\left(\alpha_{s}, \beta_{s}, \gamma_{s}\right)$ and $\left(\alpha_{f}, \beta_{f}, \gamma_{f}\right)$. Using four conditions $P_{s}, \vec{d}_{s}, P_{f}$ and $\vec{d}_{f}$, polynomials $u(h), v(h), p(h)$ and $q(h)$ are calculated which leads to the calculation of the control points as in [17]. Due to the presence of two free control points $\left(P_{2}, P_{3}\right)$, there are infinite solutions for quintic $\mathrm{PH}$ curves. Therefore an optimal solution is selected with minimum bending energy as discussed in [17] to reconstruct the shape of the CBHA manipulator. This is because a physical system always tries to move from one point to the other point with minimum potential energy (combination of bending and twisting energy).

\section{Shape IMPROVEMENTS FOR CBHA MANIPULATOR}

\section{A. Experimental Tests}

An OptiTrack vision system (Fig. 3) is used for all of the experimental validations. Four Prime13 cameras are used for tracking, each of them having specifications as follows:

- Resolution: $1.3 \mathrm{MP}(1280 \times 1024)$

- Frame rate: 240 FPS

- Filter Switcher: Included

- Interface: GigE/PoE

- No. of LEDs: 62

- Latency: $4.2 \mathrm{~ms}$

Motive 2.0 software is used for this OptiTrack vision system.

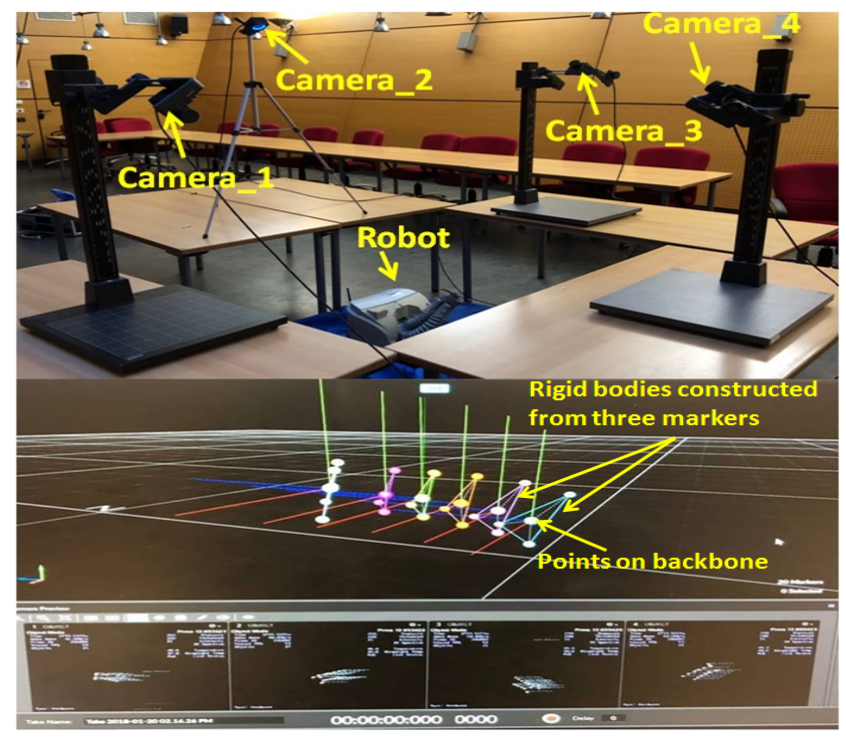

Fig. 3. Experimental Set-up to track the shape of the CBHA arm in Motive 2.0

The PH curves modeling method is applied to Compact Bionic Handling Assistant (CBHA) manipulator of Fig. 1, designed by Festo [21]. As discussed in section III, the modeling method shows how the backbone shape of the CBHA can be reconstructed by using PH-curves. To perform

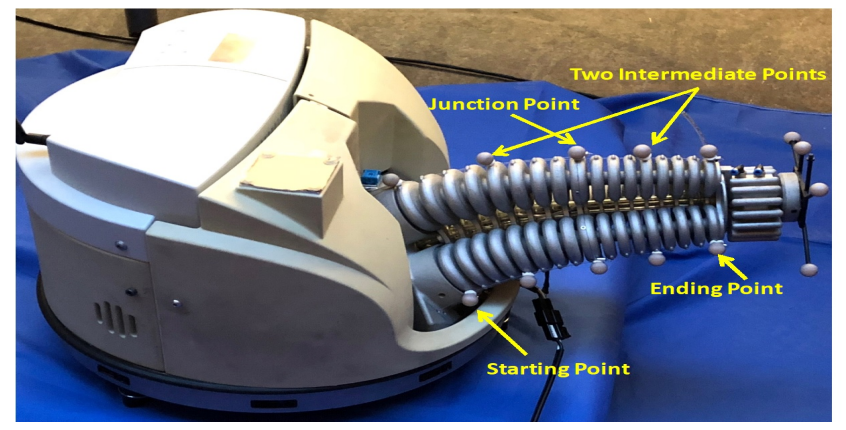

Fig. 4. Shape tracking of CBHA arm

the experimental validation, five markers are attached to each tube of the CBHA arm (at corresponding levels) (Fig. 4) 
i.e. at starting point, ending point, junction point and two intermediate points. Virtual rigid bodies are created using corresponding markers of each tube to calculate the position and orientation at corresponding backbone point (Fig. 3). Inputs are given to CBHA arm to create a random posture. Using vision system, the poses of the backbone of the CBHA, are tracked. Four inputs to construct a PH curve for CBHA arm are starting point, direction vector at the starting point, ending point and direction vector at the ending point. Therefore, a PH curve is constructed representing the shape of the CBHA arm. The junction point and the other two intermediate points are tracked to verify the reconstructed shape.

\section{B. Reforming The Shape}

When the $\mathrm{PH}$ based reconstructed shape and backbone points (indicator of actual shape) from experimental data are superimposed, a slight deviation is observed. So PH curves based approach can construct the nearest possible shape of the CBHA manipulator, but the reconstructed shape has some errors from the actual shape. These errors occur because:

- There is non-uniformity in the structure of the tubes. As, in the case of the CBHA manipulator, each tube is made up of the concatenation of 16 vertebrae. Also, the diameter of each tube decreases continuously from its base to the top.

- The material has highly non-linear elastic behavior.

Therefore, the minimum energy calculation of the PH curve does not fully adhere to these two conditions.

The reconstructed shape needs to be improved for better accuracy with minimum effort. It is observed that the reconstructed shape does not pass through the junction point of two CBHA sections. The junction point should lie on the curve defining the shape of the manipulator. Therefore, a corrected curve is generated which not only closely resembles the PH curve but is also made to pass through the junction point. Bezier interpolation is used for the initial reconstruction of the PH based shape (eq. (13)). So, the properties of bezier polynomial are exploited for generating the corrected curve. Hence, the corrected shape is also a bezier curve of the same order. This is achieved as follows:

1) The curvilinear coordinate $(h)$ of the bezier curve varies from 0 to 1 . Therefore, it is important to determine the correct value of curvilinear coordinate (h) at the junction point of two sections. As the $\mathrm{PH}$ curve gives very close initial results, it is assumed that the $\mathrm{PH}$ and the corrected curves are similar in shape. Therefore, the value of curvilinear coordinate $(h)$ of the point closest to the junction point in the $\mathrm{PH}$, is selected as the value of the curvilinear coordinate $(h)$ in the corrected shape.

2) As noted previously, we need to correct the shape with minimum effort and minimum deviation from $\mathrm{PH}$. So, in this method, only one control point is altered to shift the curve so as to pass it through the junction point. In a bezier curve, the control points have global influence, but the weight of each control point at a particular value of curvilinear coordinate $(h)$ is given by the variation in Bernstein coefficients (Fig. 5). To have a minimum deviation from the initial $\mathrm{PH}$, only the control point which has the maximum influence at the selected curvilinear coordinate $(h)$, is shifted.

3) The bezier curve eq. (12) is inverted to find the desired control point when the value of curvilinear coordinate (h) and the desired junction point is known.

$$
P_{i}=\frac{r-\sum_{k \neq i, k=0}^{5}\left(\begin{array}{l}
5 \\
k
\end{array}\right)(1-h)^{5-k} h^{k} P_{k}}{\left(\begin{array}{l}
5 \\
i
\end{array}\right)(1-h)^{5-i} h^{i}}
$$

Here, $P_{i}$ is the corrected $i_{t h}$ control point, $r$ is the desired junction point from experimental data and $h$ is the desired value of the curvilinear coordinate at the junction point.

In general, the $i_{t h}$ control point to be corrected can be selected by noticing the most influential control point from Fig. 5, corresponding to the curvilinear coordinate $(h)$ at the junction point. Experimental work space analysis of CBHA (subsection C) shows that value of $h$ ranges from 0.41 to 0.61 . Therefore, in this range the value of $i$ is either 2 or 3 (Fig. 5).

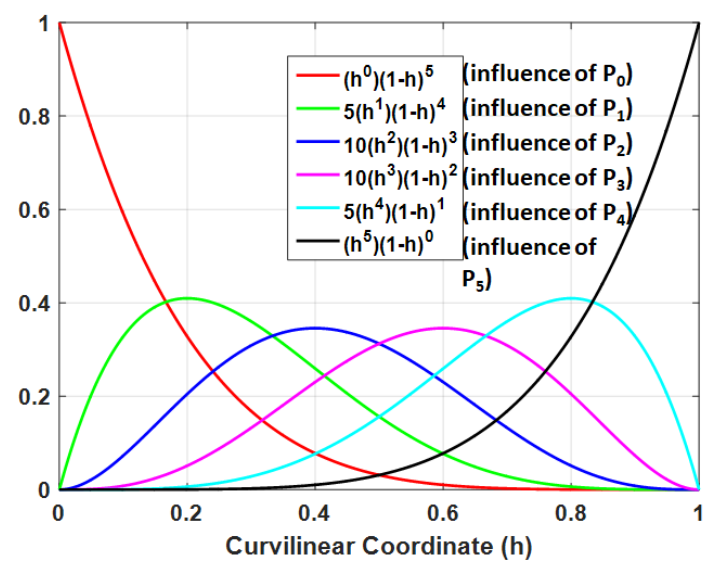

Fig. 5. Variation of Bernstein coefficients with curvilinear coordinate for quintic bezier curve

\section{Establishing Shape Relationship}

As discussed above, the new control points are computed to correct the $\mathrm{PH}$ based reconstruction. Now, it is advantageous to develop a relationship between the old conditions (old control point, $h$ corresponding to the junction point) and the new control points. This relation is developed using neural networks approach. Markers are attached on the CBHA manipulator as shown in Fig. 4. Data for all of the work space (4096 samples) of the CBHA manipulator is tracked using vision system. This data is used to compute the shapes of the backbone of the CBHA manipulator for all of the work space using PH curves based approach. The control points are recorded for all of the $\mathrm{PH}$ curves. Then the new control points are computed using the shape correction 
method as discussed above. The new control points are also recorded from the corrected curves. Therefore, a relation is developed to compute the new control points directly from the old conditions using neural networks.

Depending on the value of $h$, an NN is selected to find new control point. For $h<=0.5, P_{2}$ is recalculated as this condition implies that $P_{2}$ has maximum influence at corresponding value of $h$. Otherwise $P_{3}$ is recalculated. Fig.

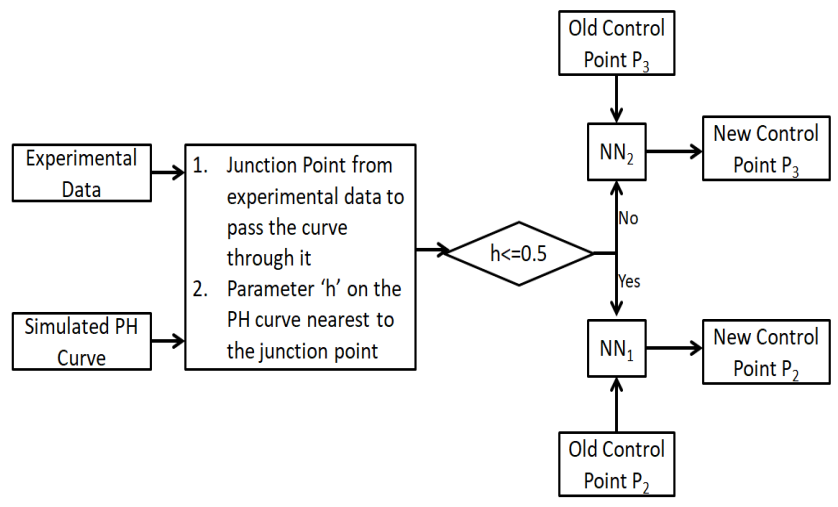

Fig. 6. Block representation of correction method

6 presents a block representation of the discussed method used to find the new control points for the corrected shape of the CBHA manipulator.

\section{Results And Discussions}

To validate the proposed method, the corrected shape is deduced by calculating the new control points. Fig. 7 presents the comparison of actual shape, $\mathrm{PH}$ based shape construction and the corrected shape using proposed methodology. Here, the three intermediate tracked marker points are at distances $1.7132 \mathrm{~mm}, 0 \mathrm{~mm}$, and $2.5424 \mathrm{~mm}$ respectively from the corrected shape. These distances are less than the distances of PH based shape $(1.6135 \mathrm{~mm}, 4.2048 \mathrm{~mm}$, and 3.8339 $\mathrm{mm})$. Therefore, an overall improvement in accuracy of the shape of CBHA manipulator is validated.

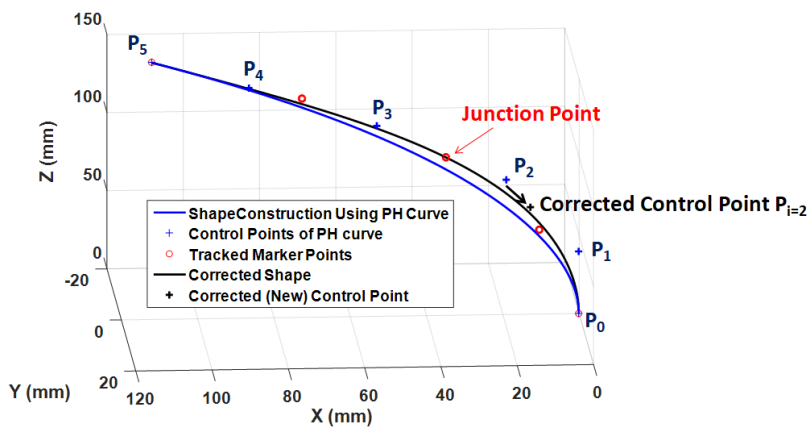

Fig. 7. Verification of the $\mathrm{PH}$ based shape reconstruction as well as corrected shape with the experimental data

Furthermore, a trajectory (Fig. 8) with 50 points is recorded using vision system. Base of the CBHA manipulator is fixed, so, the starting point as well as orientation at this point remains fixed. Ending point as well as its orientation is taken from the tracked trajectory (Fig. 8). PH curves methodology is used to reconstruct the shape of the backbone of the CBHA arm for the whole trajectory. Some random postures reconstructed using PH curves are shown in Fig. 9. The corrected shapes are also constructed using the proposed method (Fig. 9). In Fig. 9, control points of the curves are not shown to avoid the confusion with the tracked marker points. To compare, the PH based and the corrected shape with the experimental data, the distances of the junction point and two intermediate points from both of the shapes are tracked over the trajectory. The average errors for the whole trajectory (50 postures) are tabulated in Table I.

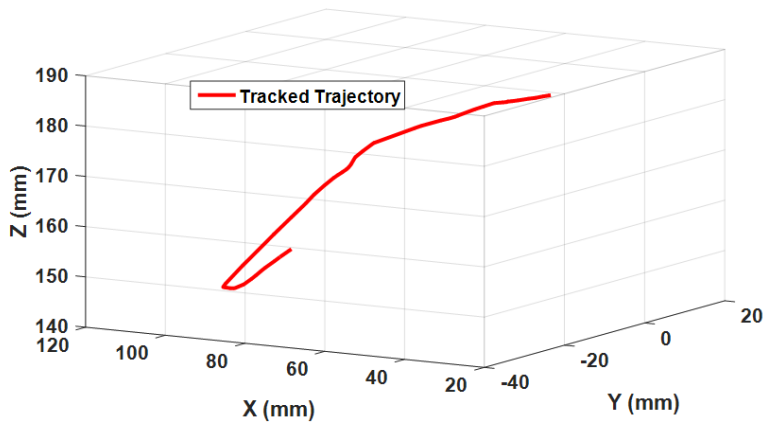

Fig. 8. Trajectory tracked using vision system

TABLE I

AVERAGE ERRORS FOR WHOLE TRAJECTORY (50 POSTURES) IN MM

\begin{tabular}{|c|c|c|}
\hline & PH based shape & Corrected Shape \\
\hline Distance from marker 2 & 1.3331 & 1.2088 \\
\hline Distance from junction point & 3.6660 & 0.0000 \\
\hline Distance from marker 4 & 4.2215 & 2.9104 \\
\hline
\end{tabular}

To establish the relationship between initial PH shape and the new control points, neural network approach is used. As already discussed, in our case either control point $P_{2}$ or $P_{3}$ is changed depending upon the value of curvilinear coordinate (h) at the junction point. This property was exploited to split the input data to train two different neural networks as shown in Fig. 6. Levenberg-Marquardt method in MATLAB is used to train both of these networks each containing ten neurons in a single hidden layer. In $N N_{1}$, the MSEs of $2.21783 \times 10^{-5}$, $3.89162 \times 10^{-5}$ and $3.60386 \times 10^{-5}$ are achieved for training, validation and test sets respectively. In $\mathrm{NN}_{2}$, the MSEs of $2.37198 \times 10^{-5}, 1.42342 \times 10^{-5}$ and $4.302266 \times 10^{-5}$ are achieved for training, validation and test sets respectively.

PH curves can approximate the shape of the continuum manipulators as stated in [17]. This $\mathrm{PH}$ based approach is applied to CBHA manipulator. But due to the non-uniform and non-linear nature of CBHA manipulator, there are some errors in the $\mathrm{PH}$ based shape construction. From table I, it is clear that these errors are reduced by using the proposed method to correct the $\mathrm{PH}$ based shape. As we tried to improve the overall errors in the shape only by changing 


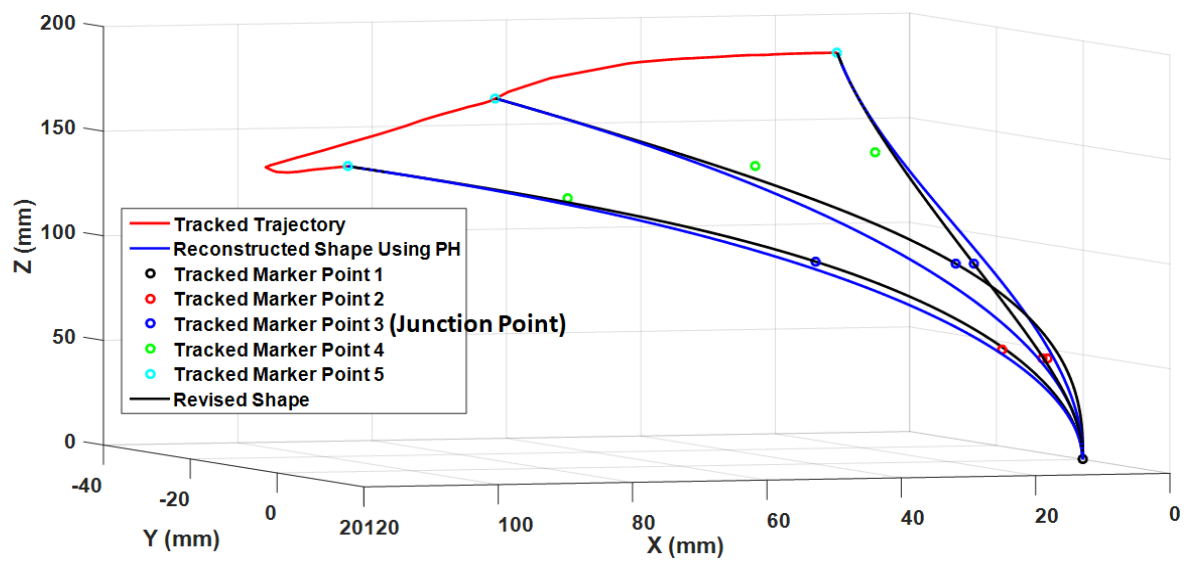

Fig. 9. Random postures reconstructed using PH curves as well as the corrected method along the trajectory

one of the existing control points of the $\mathrm{PH}$ based curve, this can be the reason for the remaining errors.

\section{CONCLUSIONS}

In this paper, a method to improve the accuracy of the shape approximation is proposed and validated experimentally. PH based shape reconstruction approach is applied to CBHA manipulator. Then the accuracy of the reconstructed shape is improved using the proposed method. Comparison with the experimental data indicates the enhancement of the performances regarding the accuracy for the same trajectory. This is a generalized method. It can be applied to any continuum manipulator to improve its $\mathrm{PH}$ based approximated shape. Our future work includes the further improvements in the shape by adjusting a combinations of control points and also applying it to the other continuum robots.

\section{ACKNOWLEDGMENT}

This work was supported by the joint Collaborative Research Project between CNRS in France and DST in India (PRC CNRS-DST 2016-2018). The authors want to thank Dr. Reinhard Pittschellis and Dr. Dirk Pensky from Festo Didactic company in Denkendorf (Germany) for their technical assistance and support on RobotinoXT.

\section{REFERENCES}

[1] R. Behrens, M. Poggendorf, E. Schulenburg, N. Elkmann. An elephant's trunk-inspired robotic arm-trajectory determination and control. In Robotics, 2012 7th German Conference: 1-5.

[2] M. Sfakiotakis, A. Kazakidi, N. Pateromichelakis, D.P. Tsakiris. Octopus-inspired eight-arm robotic swimming by sculling movements. In Robotics and Automation (ICRA), 2013 IEEE International Conference: $5155-5161$.

[3] M. Rolf, J.J. Steil. Constant curvature continuum kinematics as fas approximate model for the Bionic Handling Assistant. In Intelligent Robots and Systems (IROS), 2012 IEEE/RSJ International Conference: $3440-3446$.

[4] I. Singh, O. Lakhal, R. Merzouki. Towards Extending Forward Kinematic Models on Hyper-Redundant Manipulator to Cooperative Bionic Arms. In Journal of Physics: Conference Series. 2017; 783(1): 012056

[5] I. Singh, M. Singh, P.M. Pathak, R. Merzouki. Optimal work space of parallel continuum manipulator consisting of compact bionic handling arms. IEEE Conference on Robotics and Biomimetics (ROBIO 2017), Dec 2017, Macau, China.
[6] D. Trivedi, A. Lotfi, C.D. Rahn. Geometrically exact models for soft robotic manipulators. IEEE Transactions on Robotics. 2008; 24(4): 773-780.

[7] M. Giorelli, F. Renda, G. Ferri, C. Laschi. A feed-forward neural network learning the inverse kinetics of a soft cable-driven manipulator moving in three-dimensional space. In Intelligent Robots and Systems (IROS), 2013 IEEE/RSJ International Conference: 5033-5039.

[8] I. Singh, O. lakhal, Y. Amara, V. Coelen, P.M. Pathak, R. Merzouki. Performances evaluation of inverse kinematic models of a compact bionic handling assistant. IEEE International Conference on Robotics and Biomimetics (ROBIO). Dec, 2017: 5-8.

[9] H.A. Wurdemann, S. Sareh, A. Shafti, Y. Noh, A. Faragasso, D.S. Chathuranga, H. Liu, S. Hirai, K. Althoefer. Embedded electroconductive yarn for shape sensing of soft robotic manipulators. In Engineering in Medicine and Biology Society (EMBC), 2015 37th Annual International Conference of the IEEE: 8026-8029.

[10] H. Wang, R. Zhang, W. Chen, X. Liang, R. Pfeifer. Shape detection algorithm for soft manipulator based on fiber bragg gratings. IEEE/ASME Transactions on Mechatronics. 2016; 21(6): 2977-2982.

[11] H. Liu, A. Farvardin, R. Grupp, R.J. Murphy, R.H. Taylor, I. Iordachita, M. Armand. Shape tracking of a dexterous continuum manipulator utilizing two large deflection shape sensors. IEEE sensors journal. 2015; 15(10): 5494-5503.

[12] H. Mochiyama, E. Shimemura and H. Kobayashi. Shape correspondence between a spatial curve and a manipulator with hyper degrees of freedom. In Intelligent Robots and Systems (IROS), 1998 IEEE/RSJ International Conference: 161-166.

[13] G.S. Chirikjian, J.W. Burdick. A modal approach to hyper-redundant manipulator kinematics. IEEE Transactions on Robotics and Automation. 1994; 10(3): 343-354.

[14] R.T. Farouki, T. Sakkalis. Pythagorean hodographs. IBM Journal of Research and Development. 1990; 34(5): 736-752.

[15] M.A. Shah, N. Aouf. 3d cooperative pythagorean hodograph path planning and obstacle avoidance for multiple UAVs. In Cybernetic Intelligent Systems (CIS), 2010 IEEE 9th International Conference: $1-6$.

[16] H. Bruyninckx, D. Reynaerts. Path planning for mobile and hyperredundant robots using Pythagorean hodograph curves. In Advanced Robotics, 1997 8th International Conference: 595-600.

[17] I. Singh, Y. Amara, A. Millengui, P. M. Pathak, R. Merzouki. Modeling of continuum manipulators using pythagorean hodograph curves. Soft Robotics. Accepted.

[18] Pythagorean-hodograph curves: algebra and geometry inseparable (Vol. 1) Farouki RT; Springer Science \& Business Media: 2008.

[19] R. Dietz, J. Hoschek, B. Jttler. An algebraic approach to curve and surfaces on the sphere and on other quadrics. Computer Aided Geometric Design. 1993; 10(3-4): 211-229.

[20] R.T. Farouki, M. al-Kandari, T. Sakkalis. Hermite interpolation by rotation-invariant spatial Pythagorean-hodograph curves. Advances in Computational Mathematics. 2002; 17(4): 369-383.

[21] https://www.festo.com/group/en/cms/10239.htm. 